\title{
Metastatic Pattern of Stage IV Colorectal Cancer with High-Frequency Microsatellite Instability as a Prognostic Factor
}

\author{
KENJI FUJIYOSHI ${ }^{1,2}$, GOU YAMAMOTO ${ }^{1}$, TAKASHI TAKENOYA ${ }^{3}$, AKEMI TAKAHASHI ${ }^{1}$, \\ YOSHIKO ARAI $^{1}$, MINA YAMADA ${ }^{1}$, MIHO KAKUTA $^{1}$, KENSEI YAMAGUCHI $^{4}$, \\ YOSHITO AKAGI ${ }^{2}$, YOJI NISHIMURA ${ }^{3}$, HIROHIKO SAKAMOTO ${ }^{3}$ and KIWAMU AKAGI ${ }^{1}$ \\ ${ }^{1}$ Division of Molecular Diagnosis and Cancer Prevention, \\ ${ }^{3}$ Division of Gastroenterological Surgery, Saitama Cancer Center, Saitama, Japan; \\ ${ }^{2}$ Department of Surgery, Kurume University, Fukuoka, Japan; \\ ${ }^{4}$ Department of Gastrointestinal Oncology, \\ Cancer Institute Hospital of Japanese Foundation for Cancer Research, Tokyo, Japan
}

\begin{abstract}
Background: A recent clinical trial on the immune check-point inhibitor pembrolizumab demonstrated that microsatellite instability (MSI) is a good biomarker for response to this inhibitor. However, clinicopathological features of advanced colorectal cancer (CRC) with high-frequency MSI (MSI-H) are unclear. Patients and Methods: A total of 2,439 surgically resected CRC tissues were analyzed for MSI status, and mutational status of V-Ki-Ras2 Kirsten rat sarcoma 2 viral oncogene homolog (KRAS), neuroblastoma RAS viral oncogene homolog (NRAS) and v-Raf murine sarcoma viral oncogene homolog B (BRAF). Stage IV cases were selected, and clinical and molecular features were evaluated. Results: There was no significant survival difference observed between MSI-H CRC and microsatellite-stable (MSS) CRC in patients with stage IV disease (3.92 vs. 2.50 years; $p=0.766)$. However, hematogenous and lymphogenous metastasis-dominant CRC with MSI-H demonstrated poor prognosis, whereas peritoneal metastasis-dominant CRC with MSI-H demonstrated good prognosis, (1.33 vs. 5.2 years; $p=0.006)$. Conclusion: Prognosis of stage IV CRC with MSI-H depended on the metastatic pattern. These findings provide useful information for the adaptation of CRC immunotherapy.
\end{abstract}

This article is freely accessible online.

Correspondence to: Kiwamu Akagi, MD, Ph.D., Division of Molecular Diagnosis and Cancer Prevention, Saitama Cancer Center, 780 Komuro, Ina-machi, Kitaadachi-gun, Saitama 3620806, Japan. Tel: +81 487221111, Fax: + 81 487221129, e-mail: akagi@cancer-c.pref.saitama.jp

Key Words: Colorectal cancer, microsatellite instability, BRAF mutation, immune-checkpoint inhibitor, Lynch syndrome.
Colorectal cancer (CRC) is the third most commonly diagnosed cancer and frequent cause of cancer-related death worldwide (1). Specifically, the 5-year survival rate of patients with advanced CRC is less than $30 \%$, and further advances in chemotherapy and surgical techniques are required to improve the survival rate of patients with CRC. Recently, novel molecular-targeting drugs, such as antibody to epidermal growth factor receptor (EGFR), are based on the molecular mechanism of carcinogenesis and achieve good efficacy. However, biomarkers are often required for appropriate therapy and are predicted based on the molecular mechanism of carcinogenesis.

During carcinogenesis, genetic changes, including deletion, insertion and substitution of nucleotides, rearrangement and epigenetic changes are accumulated. At least two types of genetic changes are known: chromosomal instability (CIN) and microsatellite instability (MSI). CIN comprises of structural and numerical changes of chromosomes, whereas MSI comprises frequent mutations in the microsatellite area without obvious structural and numerical changes of chromosomes. It has been reported that in Western countries approximately $80 \%$ of CRCs exhibit CIN and $15 \%$ have MSI (2). MSI is caused by dysfunction of the mismatch repair system, which detects and repairs mismatches that occur during DNA replication $(3,4)$. CRCs with CIN and MSI demonstrate different clinicopathological features, probably because of different mechanisms of genetic changes (5).

Sporadic CRC with high-frequency MSI (MSI-H) is prone to develop in the proximal colon and in older females. Pathological characteristics are associated with the presence of tumour-infiltrating lymphocytes, a Crohn's-like lymphocytic reaction and mucinous/signet ring cell differentiation (6-9). Although CRC with MSI-H is indicative of good prognosis in stage II and III disease (10-12), several reports suggest that the prognosis of CRC with MSI-H is poorer than that of 
microsatellite-stable (MSS) CRC in stage IV $(13,14)$, and is still unclear whether this is correct or not.

In a recent study, the immune checkpoint inhibitor pembrolizumab significantly prolonged the disease-free and overall survival (OS) rates for CRC with MSI-H compared to those for CRC with MSS (15). Therefore, immune checkpoint inhibitors are highly expected to be an effective treatment for CRC with MSI-H. In this study, we evaluated the clinicopathological and molecular characteristics of stage IV CRC with MSI-H which may become potential targets of immune checkpoint inhibitor therapy.

\section{Patients and Methods}

Patients and tissue samples. The present study was conducted using 2,439 surgically resected primary CRC cases at the Saitama Cancer Center from October 1999 to March 2014. Patients who had a history of preoperative radiotherapy or chemotherapy, inflammatory bowel disease, or of familial adenomatous polyposis were excluded. Pathological staging was performed according to the tumour, node and metastasis (TNM) classification system (seventh edition) (16). Patients were followed-up until death or survival until June 2015. All tumour samples were analysed after obtaining informed consent from each patient. Tumour tissues were paired with normal colorectal tissues and stored at $-80^{\circ} \mathrm{C}$. The present study was approved by the Ethics Committee of the Saitama Cancer Center (no. 476). All procedures performed in this study were conducted in accordance with the ethical standards of the Institutional and National Research Committees and with the 1964 Helsinki Declaration and its later amendments.

Mutation analysis. Genomic DNA was extracted from the fresh frozen tissue samples using the standard phenol-chloroform extraction method and assayed for mutation in V-Ki-Ras2 Kirsten rat sarcoma 2 viral oncogene homolog (KRAS), neuroblastoma $R A S$ viral oncogene homolog (NRAS) and v-Raf murine sarcoma viral oncogene homolog B (BRAF). KRAS mutations in exon 2, 3 and 4, and NRAS mutation in exon 2 and 3 were detected by denaturing gradient gel electrophoresis or high resolution melting (HRM) analysis using a Rotor-Gene Q (Qiagen, Hilden, Germany), as described previously $(17,18)$.

$B R A F$ mutations in exon 15 (codon 600) were detected by polymerase chain reaction (PCR)-restriction fragment length polymorphism or HRM, as previously described (19).

Analysis of microsatellite status. The MSI analysis was performed using fluorescence-based PCR, as described previously (8). The MSI status was determined using five Bethesda markers: BAT25, BAT26, D5S346, D2S123 and D17S250. The MSI status was graded as MSI-H with the presence of two or more unstable markers, as MSI-low (MSI-L) with only one unstable marker, and as MSS with no unstable marker. MSI-positive markers were reexamined at least twice to confirm the results. MSI-L was grouped with MSS in this study.

Analysis of mutL homolog 1 (MLH1) promoter hypermethylation. All CRCs with MSI-H were analyzed for MLH1 promoter hypermethylation. Methylation status was investigated by methylation-specific PCR, combined with bisulfite restriction analysis, as previously reported (19).
Statistical analysis. Possible associations between each mutation and the clinicopathological parameters of CRC were assessed using the Fisher's exact test for categorical variables and an independent $t$-test for the continuous variables. For this analysis, the OS time was calculated from either the date of surgery until the date of death from CRC, or the date of the last visit at follow-up (30 June 2015). The Kaplan-Meier method was used to estimate the distribution of OS, and the log-rank test to compare the distribution of survival time. All $p$-values were calculated using a paired test and $p$-values of less than 0.05 were considered statistically significant. All statistical analyses were performed with SPSS Statistics v.20 (SPSS, Inc., IBM Corp., Armonk, NY, USA).

\section{Results}

Patient characteristics of stage IV CRC. The frequency of MSI-H was $5.9 \%$ in all cases and $3.7 \%$ in stage IV, respectively (Table I). The frequency of MSI-H was $8.9 \%$ in stage II, the highest among all the stages (Table II). KRAS mutation was detected in $42.6 \%, N R A S$ mutation in $3.1 \%$ and $B R A F$ mutation in $4.6 \%$ of all CRC specimens (Table I). Although frequency of KRAS and NRAS mutation remained unchanged in each stage, the frequency of $B R A F$ mutation increased from $3.4 \%$ at stage 0 -I to $6.3 \%$ at stage IV $(p=0.037)$ (Table II).

Clinicopathological and molecular characteristics of stage IV CRC with MSI-H. Stage IV CRCs were categorized into two groups by MSI status. Stage IV CRCs with MSI-H were more frequently associated with proximal location $(p<0.001)$, large tumour size $(p<0.001)$, undifferentiated histological features $(p<0.001)$ and mucinous components $(p=0.038)$.

Furthermore, stage IV CRC with MSI-H exhibited a different metastatic pattern compared to that with MSS. CRCs with MSI-H were more frequently associated with the presence of peritoneal dissemination $(p=0.017)$ and less frequently associated with the presence of liver metastases $(p=0.001)$. The frequency of curative resection was significantly higher in patients with MSI-H than MSS CRC $(p=0.030)$. In addition, BRAF mutation was observed significantly more frequently in MSI-H $(p=0.011)$, whereas the frequency of the KRAS and NRAS mutations were not significantly different between CRC with MSI-H and that with MSS in stage IV (Table III).

Survival analysis classified by MSI status in stage IV CRC. There was no significant survival difference observed between patients with CRC with MSI-H and those with MSS CRC in stage IV [3.92 vs. 2.50 years; hazard ratio $(\mathrm{HR})=0.892$, 95\% confidence interval $(\mathrm{CI})=0.42-1.89$, $p=0.766]$. However, the survival curve for patients with CRC with MSI-H dropped sharply, intersected with that of CRC with MSS, and was stable after 4 years (Figure 1). This 
Table I. Characteristics of patients with colorectal cancer $(C R C)$.

\begin{tabular}{|c|c|c|c|c|}
\hline & \multicolumn{2}{|c|}{ All CRC } & \multicolumn{2}{|c|}{ Stage IV } \\
\hline & $\mathrm{n}=2439$ & $(\%)$ & $\mathrm{n}=401$ & $(\%)$ \\
\hline \multicolumn{5}{|l|}{ Gender } \\
\hline Female & 1017 & $(41.7)$ & 170 & $(42.4)$ \\
\hline Male & 1422 & $(58.3)$ & 231 & $(57.6)$ \\
\hline Mean age at diagnosis $\pm \mathrm{SD}$, years & \multicolumn{2}{|c|}{$65.0 \pm 10.3$} & \multicolumn{2}{|c|}{$63.4 \pm 10.6$} \\
\hline \multicolumn{5}{|l|}{ Location } \\
\hline $\mathrm{C}$ & 192 & (7.9) & 45 & $(11.2)$ \\
\hline A & 317 & $(13.0)$ & 64 & $(16.0)$ \\
\hline $\mathrm{T}$ & 216 & $(8.9)$ & 37 & $(9.2)$ \\
\hline $\mathrm{D}$ & 76 & $(3.1)$ & 10 & $(2.5)$ \\
\hline $\mathrm{S}$ & 622 & $(25.5)$ & 111 & $(27.7)$ \\
\hline $\mathrm{RS}$ & 313 & $(12.8)$ & 54 & $(13.5)$ \\
\hline $\mathrm{Ra} \cdot \mathrm{Rb} \bullet \mathrm{P}$ & 703 & $(28.8)$ & 80 & $(19.9)$ \\
\hline \multicolumn{5}{|l|}{ Location } \\
\hline Proximal & 725 & $(29.7)$ & 146 & $(36.4)$ \\
\hline Distal & 1714 & $(70.3)$ & 255 & $(63.6)$ \\
\hline Mean tumor size $\pm \mathrm{SD}, \mathrm{mm}$ & \multicolumn{2}{|c|}{$45.6 \pm 23.8$} & \multicolumn{2}{|c|}{$60.4 \pm 26.6$} \\
\hline \multicolumn{5}{|l|}{ TNM stage } \\
\hline $0-\mathrm{I}$ & 546 & $(22.4)$ & - & - \\
\hline II & 762 & $(31.2)$ & - & - \\
\hline III & 730 & $(29.9)$ & - & - \\
\hline IV & 401 & (16.4) & 401 & $(100)$ \\
\hline \multicolumn{5}{|l|}{ Histological feature } \\
\hline Well & 218 & $(8.9)$ & 10 & $(2.5)$ \\
\hline Moderate & 2064 & $(84.6)$ & 345 & $(86.0)$ \\
\hline Poor & 74 & $(3.0)$ & 21 & $(5.2)$ \\
\hline Mucinous & 82 & (3.4) & 25 & (6.2) \\
\hline Signet & 1 & $(0.1)$ & 0 & $(0.0)$ \\
\hline \multicolumn{5}{|l|}{ Mucinous component } \\
\hline No & 2136 & $(87.6)$ & 328 & $(81.8)$ \\
\hline Yes & 303 & $(12.4)$ & 73 & $(18.2)$ \\
\hline \multicolumn{5}{|l|}{ MSI status } \\
\hline MSS & 2295 & $(94.1)$ & 386 & $(96.3)$ \\
\hline MSI-H & 144 & $(5.9)$ & 15 & (3.7) \\
\hline \multicolumn{5}{|l|}{ KRAS } \\
\hline Wild-type & 1369 & $(57.4)$ & 211 & $(53.3)$ \\
\hline Mutant & 1016 & (42.6) & 185 & $(46.7)$ \\
\hline ND & 54 & - & 5 & - \\
\hline \multicolumn{5}{|l|}{$N R A S$} \\
\hline Wild-type & 2313 & (96.9) & 381 & (96.7) \\
\hline Mutant & 73 & (3.1) & 13 & (3.3) \\
\hline ND & 53 & - & 7 & - \\
\hline \multicolumn{5}{|l|}{$B R A F$} \\
\hline Wild-type & 2280 & (95.4) & 371 & (93.7) \\
\hline Mutant & 109 & (4.6) & 25 & (6.3) \\
\hline ND & 50 & - & 5 & - \\
\hline
\end{tabular}

SD: Standard deviation, C: cecum, A: ascending colon, T: transverse colon, D: descending colon, S: sigmoidal colon, RS: rectosigmoid, Ra: rectum above the peritoneal reflexion, $\mathrm{Rb}$ : rectum below the peritoneal reflexion, $\mathrm{P}$ : proctos, Proximal: cecum to transverse colon, Distal: splenic flexure to sigmoidal colon, TNM: tumor-node-metastasis, well: well-differentiated tubular adenocarcinoma, moderate: moderately differentiated tubular adenocarcinoma, poor: poorly differentiated tubular adenocarcinoma, mucinous: mucinous adenocarcinoma, signet: signet ring cell carcinoma, ND: not determined.

suggests that there are two groups in CRC with MSI-H: one with poor prognosis and another with good prognosis. Univariate and multivariate survival analyses for CRC with MSI-H demonstrated that there was no statistical significance, due to a small number of MSI-H cases. However, it did suggest that the metastatic pattern (liver metastasis- or peritoneal metastasis-dominant) was associated with survival in CRC with MSI-H (Table V). 
Table II. Rates for mutation (mt) of v-Raf murine sarcoma viral oncogene homolog B (BRAF), V-Ki-Ras2 Kirsten rat sarcoma 2 viral oncogene homolog (KRAS) and neuroblastoma RAS viral oncogene homolog (NRAS), and for high-frequency microsatellite instability (MSI$H)$ by colorectal cancer stage.

\begin{tabular}{ccccc}
\hline & BRAF $\mathrm{mt}(\%)$ & KRAS $\mathrm{mt}(\%)$ & NRAS mt (\%) & MSI-H (\%) \\
\hline Stage & & & & \\
0-I & 3.4 & 39.1 & 2.4 & 5.9 \\
II & 4.6 & 42.8 & 3.6 & 8.9 \\
III & 4.5 & 42.7 & 2.8 & 4.0 \\
IV & 6.3 & 46.7 & 3.3 & 3.7 \\
Total & 4.6 & 42.6 & 3.3 & 5.9 \\
\hline
\end{tabular}

Survival analysis of patients with stage IV CRC classified by metastatic pattern and MSI status. We divided patients with stage IV CRC with MSI-H and MSS into two groups: i) a CRC with peritoneal metastasis-dominant group (PM); and ii) a CRC with hematogenous and lymphogenous metastasis-dominant group (HL). CRC with PM was defined as cases with peritoneal metastasis with fewer than four lymph node metastases. CRC with HL which included CRC with liver metastasis, lung metastasis, or more than three lymph node metastases was defined. Finally, stage IV CRCs were classified into four groups: i) MSI-H CRC with HL (MSI-H-HL); ii) MSI-H CRC with PM (MSI-MSI-H-PM); iii) MSS CRC with HL (MSS-HL); and iv) MSS CRC with PM (MSS-PM).

Patients with MSI-H-HL had the poorest OS rate of the four groups (vs. MSI-H-PM, $p=0.006$; vs. MSS-PM, $p=0.004 ; v s$. MSS-HL, $p=0.021)$. In the multivariate analysis, there was a significant decrease in the median OS rate for those with MSI-H-HL compared to those with MSSHL (1.33 vs. 2.42 years; $\mathrm{HR}=2.34,95 \% \mathrm{CI}=1.01-4.99$; $p=0.027)$ and MSS-PM (1.33 vs. 5 years; $\mathrm{HR}=4.03,95 \%$ $\mathrm{CI}=1.47-11.04 ; p=0.007)$. In contrast, all patients survived in the MSI-H-PM group, which demonstrated the best OS rate of the four groups (vs. MSI-H-HL, $p=0.006$; vs. MSSPM, $p=0.08 ; v s$. MSS-HL, $p=0.022$ ) (Figure 2).

Pathological and molecular characteristics of MSI-H-PM and $M S I-H-H L$. The frequency of KRAS mutation between MSIH-PM and MSI-H-HL groups was not different $(p=1.000)$. In contrast, the frequency of $B R A F$ mutation in MSI-H-HL cases $(40 \%)$ tended to be higher than that in MSI-H-PM cases $(0 \%)$ ( $p=0.231$ ), no MSI-H-PM case had $B R A F$ mutation.

$B R A F$-mutated CRC with MSI-H demonstrated a poorer prognosis than wild-type $B R A F \mathrm{CRC}$ with MSI-H in stage IV $(\mathrm{HR}=1.575, \mathrm{CI}=0.286-8.673 ; p=0.601 v s . B R A F$ wildtype) (Figure 3). MLH1 promoter hypermethylation was observed in eight cases among 15 stage IV CRCs with MSI$\mathrm{H}$, consisting of two MSI-H-PM and six MSI-H-HL cases

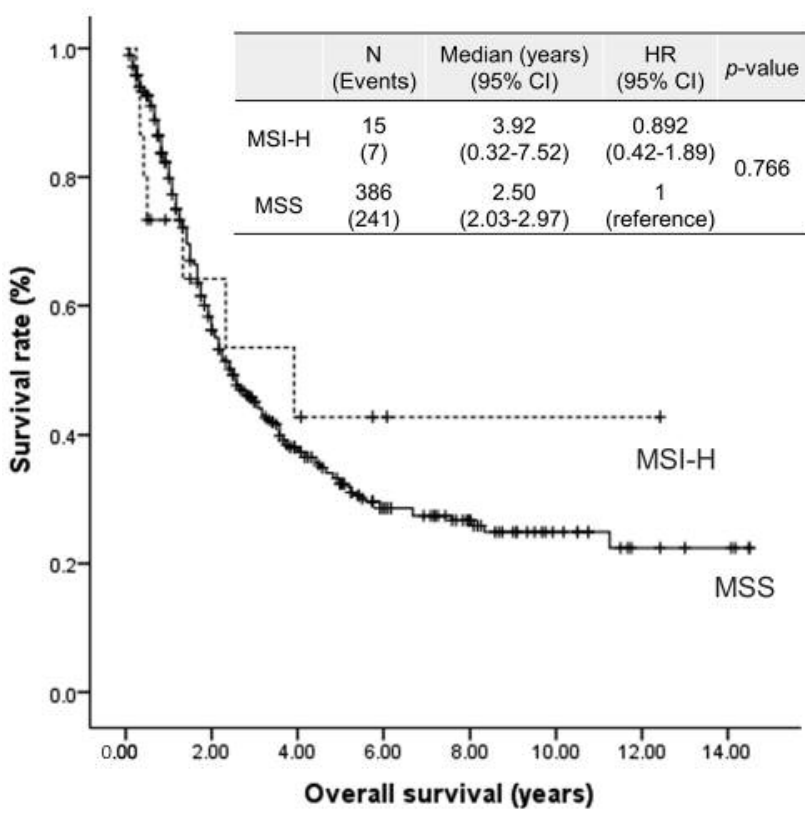

Figure 1. Survival analysis of patients with stage IV colorectal cancer classified by microsatellite instability (MSI) status. There was no significant survival difference for those with high-frequency MSI (MSIH) CRC compared to those with microsatellite stable (MSS) CRC. HR: Hazard ratio, CI: confidence interval.

(Table IV). OS rates were not significantly different between hypermethylated MLHI promoter CRC with MSI-H and unmethylated CRC with MSI-H in stage IV (Figure 4).

In comparison between MSI-H and MSS, the frequency of $B R A F$ mutation in MSI-H-HL cases was significantly higher than that in MSS-HL cases $(p=0.002)$. Moreover, the curability rate of MSI-H-HL tended to be higher than MSS-HL $(p=0.120)$. The curability rate of MSS-PM was significantly higher than that of MSS-HL $(p=0.003)$ (Table IV).

\section{Discussion}

In this study, we found that there were two main groups in stage IV CRC with MSI-H, one with extremely good prognosis (MSI-H-PM), and one with a poor prognosis (MSI-H-HL). In general, CRC with MSI-H is a better prognostic indicator than MSS in stage II and III CRC (1012), whereas no difference was observed between corresponding groups in stage IV CRC in our study (Figure 1). However, the Kaplan-Meier curve in Figure 1 indicates that stage IV CRC with MSI-H may consist of two different groups: i) a group with poor prognosis, demonstrated by the curve dropping sharply during the early phase; and ii) a group with long-term survival. The univariate and multivariate analyses suggest that the metastatic patterns do indeed affect prognosis (Table V). Several reasons can be 
Table III. Characteristics of patients with stage IV colorectal cancer.

\begin{tabular}{|c|c|c|c|c|c|c|c|}
\hline & \multicolumn{3}{|c|}{ MSI-H } & \multicolumn{3}{|c|}{ MSS } & \multirow[t]{2}{*}{$p$-Value } \\
\hline & $\mathrm{n}=15$ & & $(\%)$ & $\mathrm{n}=386$ & & $(\%)$ & \\
\hline \multicolumn{8}{|l|}{ Gender } \\
\hline Female & 9 & & $(60.0)$ & 161 & & $(41.7)$ & $0.188^{\dagger}$ \\
\hline Male & 6 & & $(40.0)$ & 225 & & $(58.3)$ & \\
\hline Mean age at diagnosis $\pm \mathrm{SD}$, years & & $60.0 \pm 17.5$ & & & $63.5 \pm 10.2$ & & $0.424 \ddagger$ \\
\hline \multicolumn{8}{|l|}{ Location } \\
\hline $\mathrm{C}$ & 2 & & (13.3) & 43 & & $(11.1)$ & $0.017^{\dagger}$ \\
\hline A & 7 & & (46.7) & 57 & & $(14.8)$ & \\
\hline $\mathrm{T}$ & 3 & & $(20.0)$ & 34 & & $(8.8)$ & \\
\hline $\mathrm{D}$ & 1 & & $(6.7)$ & 9 & & $(2.3)$ & \\
\hline $\mathrm{S}$ & 1 & & (6.7) & 110 & & $(28.5)$ & \\
\hline $\mathrm{RS}$ & 0 & & $(0.0)$ & 54 & & $(14.0)$ & \\
\hline $\mathrm{Ra} \cdot \mathrm{Rb} \bullet \mathrm{P}$ & 1 & & (6.7) & 79 & & $(20.5)$ & \\
\hline \multicolumn{8}{|l|}{ Location } \\
\hline Proximal & 12 & & $(80.0)$ & 134 & & $(34.7)$ & $<0.001^{\dagger}$ \\
\hline Distal & 3 & & $(20.0)$ & 252 & & $(65.3)$ & \\
\hline Mean tumor size $\pm \mathrm{SD}, \mathrm{mm}$ & & $89.0 \pm 36.5$ & & & $59.3 \pm 25.5$ & & $<0.001^{\ddagger}$ \\
\hline \multicolumn{8}{|l|}{ Histological feature } \\
\hline Well & 0 & & $(0.0)$ & 10 & & (2.6) & $<0.001^{\dagger}$ \\
\hline Moderate & 8 & & $(53.3)$ & 337 & & $(87.3)$ & \\
\hline Poor & 5 & & (33.3) & 16 & & $(4.1)$ & \\
\hline Mucinous & 2 & & (13.3) & 23 & & $(6.0)$ & \\
\hline Signet & 0 & & $(0.0)$ & 0 & & $(0.0)$ & \\
\hline \multicolumn{8}{|l|}{ Mucinous component } \\
\hline No & 9 & & $(60.0)$ & 319 & & $(82.6)$ & $0.038^{\dagger}$ \\
\hline Yes & 6 & & $(40.0)$ & 67 & & $(17.4)$ & \\
\hline \multicolumn{8}{|l|}{ Nodal metastases } \\
\hline $\mathrm{N} \leq 3$ & 10 & & $(66.7)$ & 235 & & $(60.9)$ & $0.790^{\dagger}$ \\
\hline $\mathrm{N} \geq 4$ & 5 & & (33.3) & 151 & & (39.1) & \\
\hline \multicolumn{8}{|l|}{ Lung metastases } \\
\hline No & 15 & & $(100.0)$ & 320 & & $(82.9)$ & $0.146^{\dagger}$ \\
\hline Yes & 0 & & $(0.0)$ & 66 & & $(17.1)$ & \\
\hline \multicolumn{8}{|l|}{ Liver metastases } \\
\hline No & 11 & & $(73.3)$ & 112 & & $(29.0)$ & $0.001^{\dagger}$ \\
\hline Yes & 4 & & (26.7) & 274 & & $(71.0)$ & \\
\hline \multicolumn{8}{|l|}{ Peritoneal metastases } \\
\hline No & 6 & & $(40.0)$ & 276 & & $(71.5)$ & $0.017^{\dagger}$ \\
\hline Yes & 9 & & $(60.0)$ & 110 & & (28.5) & \\
\hline P1 & (4) & & (44.4) & (46) & & $(41.8)$ & \\
\hline $\mathrm{P} 2$ & (5) & & $(55.6)$ & (35) & & $(31.8)$ & \\
\hline $\mathrm{P} 3$ & (0) & & $(0.0)$ & (29) & & (26.4) & \\
\hline \multicolumn{8}{|l|}{ Curability } \\
\hline R0 & 13 & & (86.7) & 216 & & $(56.0)$ & $0.030^{\dagger}$ \\
\hline $\mathrm{R} 1$ & 2 & & (13.3) & 170 & & $(44.0)$ & \\
\hline \multicolumn{8}{|l|}{$K R A S$} \\
\hline Wild-type & 10 & & (66.7) & 201 & & (51.9) & $0.307^{\dagger}$ \\
\hline Mutant & 5 & & (33.3) & 180 & & (48.1) & \\
\hline ND & 0 & & - & 5 & & - & \\
\hline \multicolumn{8}{|l|}{$N R A S$} \\
\hline Wild-type & 15 & & $(100.0)$ & 366 & & (96.6) & $1.00^{\dagger}$ \\
\hline Mutant & 0 & & $(0.0)$ & 13 & & (3.4) & \\
\hline ND & 0 & & - & 7 & & - & \\
\hline \multicolumn{8}{|l|}{$B R A F$} \\
\hline Wild-type & 11 & & (73.3) & 360 & & $(94.5)$ & $0.011^{\dagger}$ \\
\hline Mutant & 4 & & (26.7) & 21 & & $(5.5)$ & \\
\hline ND & 0 & & - & 5 & & - & \\
\hline
\end{tabular}

MSI-H: High-frequency microsatellite instability; MSS: microsatellite stable; SD: standard deviation, C: cecum, A: ascending colon, T: transverse colon, D: descending colon, S: sigmoidal colon, RS: rectosigmoid, Ra: rectum above the peritoneal reflexion, Rb: rectum below the peritoneal reflexion, P: proctos, Proximal: cecum to transverse colon, Distal: splenic flexure to sigmoidal colon, well: well-differentiated tubular adenocarcinoma, moderate: moderately differentiated tubular adenocarcinoma, poor: poorly differenciated tubular adenocarcinoma, signet: signet ring cell carcinoma, mucinous: mucinous adenocarcinoma, P1: presence of nodules at proximal peritoneum, P2: few nodules at distal peritoneum, P3: many nodules at distal peritonum, R0: no cancer remnant, R1: presence of cancer remnant, BRAF: v-Raf murine sarcoma viral oncogene homolog B, KRAS: V-Ki-Ras2 Kirsten rat sarcoma

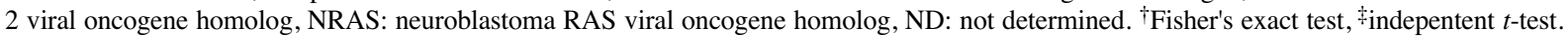


Table IV. Molecular and pathological features of each type of metastasis according to microsatellite instability (MSI) status in colorectal cancer (CRC).

\begin{tabular}{|c|c|c|c|c|c|c|c|c|}
\hline & \multicolumn{4}{|c|}{ MSI-H } & \multicolumn{4}{|c|}{ MSS } \\
\hline & \multicolumn{2}{|c|}{ MSI-H-PM } & \multicolumn{2}{|c|}{ MSI-H-HL } & \multicolumn{2}{|c|}{ MSS-PM } & \multicolumn{2}{|c|}{ MSS-HL } \\
\hline & $\%$ & (mt/Total) & $\%$ & (mt/Total) & $\%$ & (mt/Total) & $\%$ & (mt/Total) \\
\hline$K R A S$-mutant & 40.0 & $(2 / 5)$ & 30.0 & $(3 / 10)$ & 48.0 & $(12 / 25)$ & $47.2^{\mathrm{a}}$ & $(168 / 356)$ \\
\hline$N R A S$-mutant & 0.0 & $(0 / 5)$ & 0.0 & $(0 / 10)$ & 8.3 & $(2 / 24)$ & 2.9 & $(11 / 355)$ \\
\hline$B R A F$-mutant & 0.0 & $(0 / 5)$ & $40.0^{\mathrm{b}}$ & $(4 / 10)$ & $12.0^{\mathrm{c}}$ & $(3 / 25)$ & 5.1 & $(18 / 356)$ \\
\hline MLH1 methylation & 40.0 & $(2 / 5)$ & 60.0 & $(6 / 10)$ & - & - & - & - \\
\hline Curability (R0) & 100.0 & $(5 / 5)$ & $80.0^{\mathrm{d}}$ & $(8 / 10)$ & 84.0 & $(21 / 25)$ & $54.0^{\mathrm{e}}$ & $(195 / 361)$ \\
\hline
\end{tabular}

BRAF: v-Raf murine sarcoma viral oncogene homolog B, KRAS: V-Ki-Ras2 Kirsten rat sarcoma 2 viral oncogene homolog, NRAS: neuroblastoma RAS viral oncogene homolog, MLH1: mutL homolog 1, MLH1 methylation: MLH1 promoter hypermethylation, R0: no cancer remnant, MSS: microsatellite stable, MSI-H: high-frequency microsatellite instability, PM: peritoneal metastasis-dominant CRC, HL: hematogenous and lymphogenous metastasis-dominant CRC. ${ }^{\mathrm{a}} v s$. MSS-PM: $p=0.835$, $v s$. MSI-H-HL: $p=0.347 ;{ }^{\mathrm{b}} v$. MSS-HL: $p=0.002, v s$. MSS-PM: $p=0.231$; ${ }^{c} v s$. MSS-HL: $p=0.156 ;{ }^{\mathrm{d}} v$. MSI-H-PM: $p=0.524$, $v s$. MSS-HL $p=0.120, \mathrm{e}_{v s}$. MSS-PM: $p=0.003$.

Table V. Univariate and multivariate overall-survival analysis in stage IV colorectal cancer with high-frequency microsatellite instability.

\begin{tabular}{|c|c|c|c|c|c|c|}
\hline & \multicolumn{3}{|c|}{ Univariate } & \multicolumn{3}{|c|}{ Multivariate } \\
\hline & HR & $95 \% \mathrm{CI}$ & $p$-Value & HR & $95 \% \mathrm{CI}$ & $p$-Value \\
\hline \multicolumn{7}{|l|}{ Location } \\
\hline Proximal & 36.173 & $0.033-39110$ & 0.314 & - & - & - \\
\hline Distal & 1 & - & - & & & \\
\hline \multicolumn{7}{|c|}{ Mucinous component } \\
\hline Yes & 0.871 & $0.296-1.55$ & 0.358 & - & - & - \\
\hline No & 1 & - & - & & & \\
\hline \multicolumn{7}{|c|}{ Liver metastases } \\
\hline Yes & 3.378 & $0.744-15.38$ & 0.115 & 0.066 & $0.002-2.380$ & 0.137 \\
\hline No & 1 & - & - & & & \\
\hline \multicolumn{7}{|l|}{ PM } \\
\hline Yes & 1.011 & $0.191-5.347$ & 0.99 & - & - & - \\
\hline No & 1 & - & - & & & \\
\hline \multicolumn{7}{|l|}{ PM-dominant } \\
\hline Yes & 0.011 & 2.14E-5-5.681 & 0.157 & 193512 & $0.000-4.357 \mathrm{E}+269$ & 0.969 \\
\hline No & 1 & - & - & & & \\
\hline \multicolumn{7}{|l|}{ Curability } \\
\hline $\mathrm{R} 1$ & 1.904 & $0.197-18.18$ & 0.578 & - & - & - \\
\hline R0 & 1 & - & - & & & \\
\hline \multicolumn{7}{|l|}{$K R A S$} \\
\hline Mutant & 0.847 & $0.163-4.385$ & 0.843 & - & - & - \\
\hline Wild-type & 1 & - & - & & & \\
\hline \multicolumn{7}{|l|}{$B R A F$} \\
\hline Mutant & 1.574 & $0.286-8.695$ & 0.601 & - & - & - \\
\hline Wild-type & 1 & 0 & - & & & \\
\hline
\end{tabular}

HR: Hazard ratio, CI: confidence interval, proximal: cecum to transverse colon, distal: splenic flexure to sigmoidal colon, PM: peritoneal metastases, R0: no cancer remnant, R1: presence of cancer remnant, KRAS: V-Ki-Ras2 Kirsten rat sarcoma 2 viral oncogene homolog, BRAF: v-Raf murine sarcoma viral oncogene homolog B.

considered for why MSI-H-HL in stage IV CRC was found to be associated with a poor prognosis. Firstly, $B R A F$ mutation would adversely affect prognosis. It has been reported that $B R A F$ mutation was a poor prognostic factor in several retrospective studies (8, 20-22). Additionally, in CRC with MSI-H, $B R A F$-mutated CRC was also found to have poorer prognosis than CRC with wild-type BRAF (23). Similarly to these previous reports, our data show that 


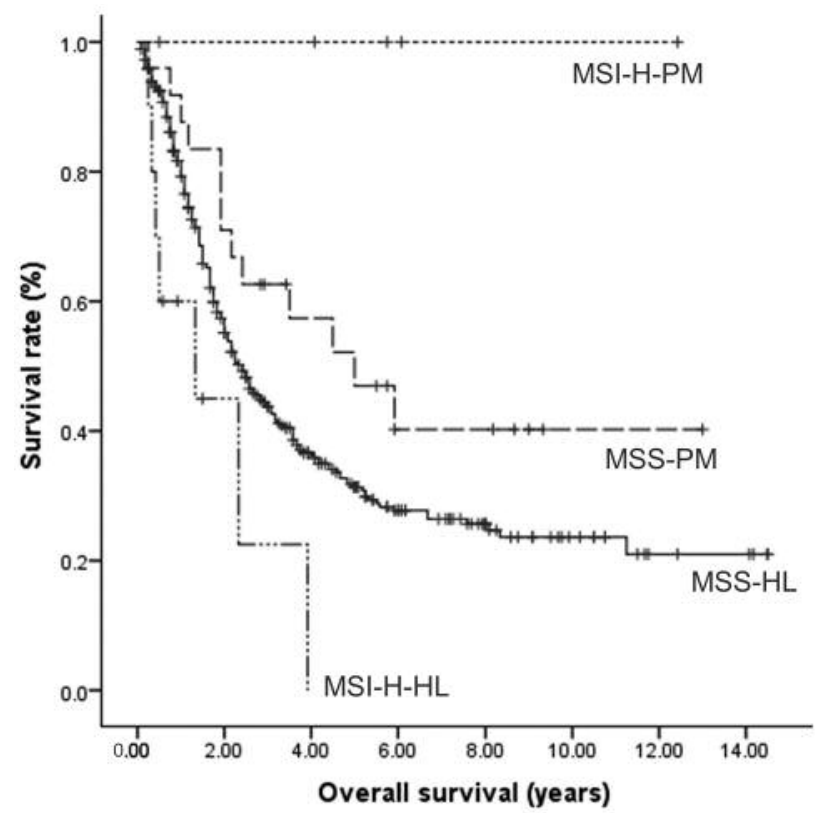

\begin{tabular}{ccccc}
\hline & $\begin{array}{c}\mathrm{N} \\
(\text { Events) }\end{array}$ & $\begin{array}{c}\text { Median (years) } \\
(95 \% \mathrm{Cl})\end{array}$ & $\begin{array}{c}\mathrm{HR} \\
(95 \% \mathrm{Cl})\end{array}$ & $p$-value \\
\hline MSI-H-HL & 10 & 1.33 & 2.34 & 0.027 \\
& $(7)$ & $(0.00-3.22)$ & $(1.01-4.99)$ & \\
MSI-H-PM & 5 & 5.20 & $\mathrm{~N} / \mathrm{A}$ & $\mathrm{N} / \mathrm{A}$ \\
& $(0)$ & $\mathrm{N} / \mathrm{A}$ & & \\
MSS-HL & 361 & 2.42 & 1 & - \\
& $(228)$ & $(0.25-13.06)$ & $($ reference) & - \\
MSS-PM & 25 & 5.00 & 0.60 & 0.076 \\
& $(13)$ & $(0.12-14.56)$ & $(0.34-1.05)$ & 0.07 \\
\hline
\end{tabular}

Figure 2. Survival analysis of patients with stage IV colorectal cancer CRC classified by metastatic pattern and microsatellite instability (MSI) status. Those with hematogenous/lymphogenous metastasis-dominant MSI-high (MSI-H-HL) CRC had the poorest overall survival out of the four groups [vs. peritoneal metastasis-dominant (MSI-H-PM): $p=0.006$; vs. microsatellite stable (MSS): $p=0.004 ; v s$. MSS-HL: $p=0.021]$. All cases in the MSI-H-PM group survived and MSI-H-PM had the best overall survival rate out of the four groups (vs. MSI-H-HL: $p=0.006$; vs. MSS-PM: $p=0.08$; vs. MSS-HL: $p=0.022)$. HR: Hazard ratio, CI: confidence interval.

$B R A F$-mutated stage IV CRC with MSI-H demonstrated a poorer prognosis than that wild-type $B R A F$. Whereas, $B R A F$ mutation with MSI-H was not associated with poorer OS than BRAF mutation with MSS in stage I-III CRC (24). Poor prognosis associated with $B R A F$ mutation may be dependent on tumour stage. Furthermore, the frequency of $B R A F$ mutation was $40 \%$ in MSI-H-HL compared to none in MSIH-PM cases. Since not all cases of MSI-H-HL had the BRAF mutation, there may be factors of poor prognosis at play other than the $B R A F$ mutation. In the future, we plan to investigate the mechanism of this association by

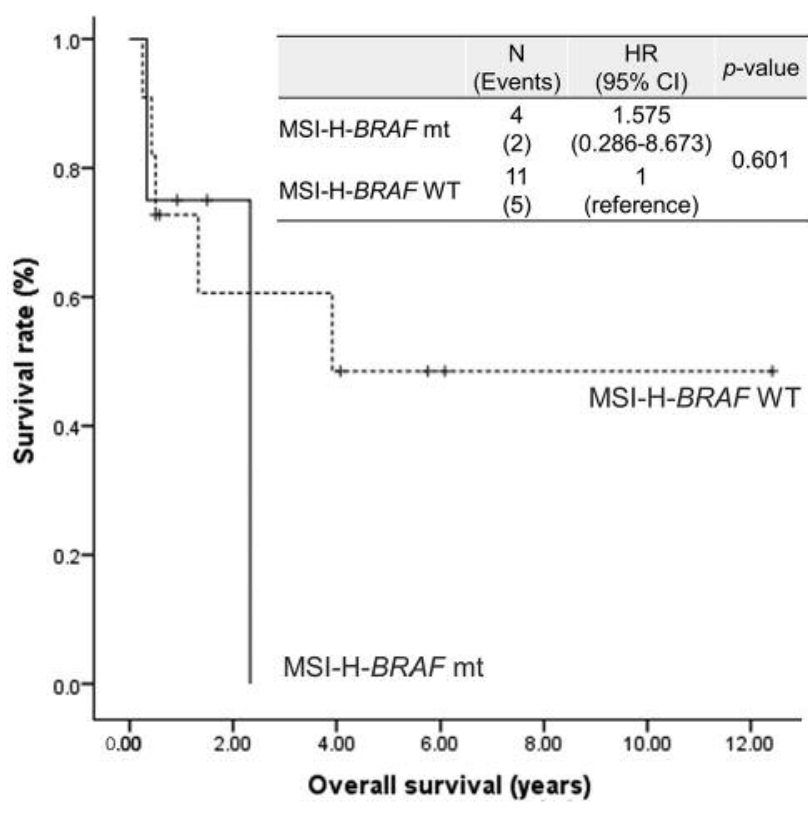

Figure 3. Survival analysis of patients with stage IV colorectal cancer classified by $v$-Raf murine sarcoma viral oncogene homolog $B$ (BRAF) mutation. Patients with BRAF mutation (mt) had a poorer prognosis compared to those with wild-type (WT) BRAF. HR: Hazard ratio, CI: confidence interval.

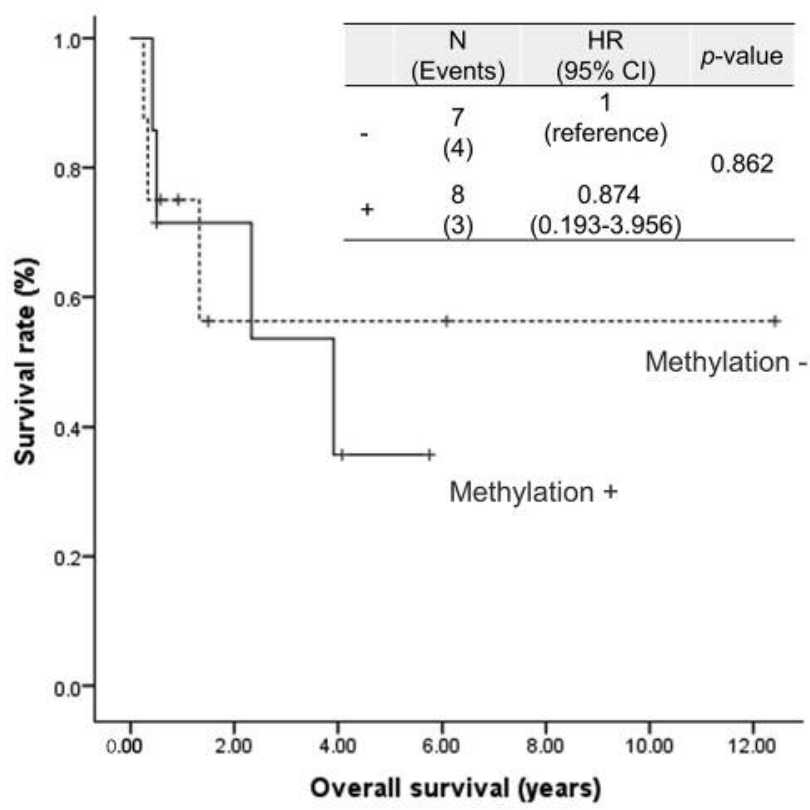

Figure 4. Survival analysis of patients with stage IV colorectal cancer classified by the methylation status of the mutL homolog 1 (MLH1) promoter. Survival of patients with hypermethylation of the MLH1 promoter $(+)$ was not significantly different from that of those without MLH1 promoter hypermethylation (-). HR: Hazard ratio, CI: confidence interval. 
accumulating more cases and examining multiple cancerrelated genes.

In general, CRC with MSI-H exhibits a large tumour size and has high growth ability but less metastasis $(18,25,26)$. Therefore, once these tumours acquire metastatic potential, the prognosis might worsen. From a recent large-scale genome analysis, Guinney et al. showed there to be four consensus molecular subtypes (CMS) of CRC (27). From this study, the CMS1 population, which tended to have hypermutated type (high number of somatic mutation events in the genome) and $B R A F$ mutation, had a very poor survival rate after relapse, but good OS and relapse-free survival. A part of the CMS3 population tended to have hypermutated type and KRAS mutation. MSI-H-HL and MSI-H-PM in our study may correspond to the CMS1 and CMS3 populations, respectively.

In contrast with the poor prognosis of MSI-H-HL CRC, patients with MSI-H-PM CRC had a prolonged OS rate, and there were no deaths during the observation period. Vatandoust et al. reported that patients with PM CRC who underwent curative surgery could expect long-term survival (28). Similarly to Vatandoust et al.'s report, MSI-H-PM also conferred a better prognosis in patients with curatively resected stage IV CRC in our study.

The following are considered to be the reasons for why MSI-H-PM in stage IV CRC has a good prognosis. Firstly, MSI-H-PM cases did not have $B R A F$ mutation, which is a poor prognostic factor. Even though $B R A F$-mutated CRC is likely to develop PM (29), no MSI-H-PM cases were $B R A F$-mutant. Moreover, the report that $B R A F$-mutated CRCs with MSI-H are less likely to exhibit peritoneal metastasis supports our results (30). Thus, our detailed analysis suggests that the frequency of $B R A F$ mutations in $\mathrm{PM}$ is dependent on the MSI status. Additionally, curatively resected MSI-H-PM cases could be considered as having the same oncological status as during the early stage. Therefore, MSI-H-PM cases may maintain a better prognosis.

In this study, a multivariate analysis could not be performed sufficiently due to the small number of CRC cases with MSI-H. Frequency of CRC with MSI-H is approximately $10-15 \%$ in Western countries, whereas that in Asia was reported to be $5.5-7.3 \%(18,31,32)$, lower than in Western countries. However, despite the small number of cases, there was a significant prognostic difference between MSI-H-HL and MSI-H-PM cases. In a recent clinical trial, the immune checkpoint inhibitor, pembrolizumab prolonged disease-free and OS rates for metastatic CRC with MSI-H (15). Thus, MSI-H is an attractive biomarker for immunotherapy and these findings provide useful information for the adaptation of CRC immunotherapy. In conclusion, we found that the difference in metastatic pattern in stage IV CRC with MSI-H affects prognosis.

\section{Compliance with Ethical Standards}

This study was approved by the Ethics Committee of the Saitama Cancer Center.

\section{Conflicts of Interest}

The Authors declare that they have no competing of interest in regard to this study.

\section{Acknowledgements}

The Authors would like to thank the staff of the Divisions of Gastroenterological Surgery and Molecular Diagnosis and Cancer Prevention, Saitama Cancer Center.

\section{References}

1 Torre LA, Bray F, Siegel RL, Ferlay J, Lortet-Tieulent J and Jemal A: Global cancer statistics, 2012. CA Cancer J Clin 65: 87-108, 2015.

2 Umar A, Boland CR, Terdiman JP, Syngal S, Chapelle Adl, Ruschoff J, Fishel R, Lindor NM, Burgart LJ, Hamelin R, Hamilton SR, Hiatt RA, Jass J, Lindblom A, Lynch HT, Peltomaki P, Ramsey SD, Rodriguez-Bigas MA, Vasen HFA, Hawk ET, Barrett JC, Freedman AN and Srivastava S: Revised Bethesda Guidelines for Hereditary Nonpolyposis Colorectal Cancer (Lynch Syndrome) and Microsatellite Instability. J Natl Cancer Inst 96: 261-268, 2004.

3 Sinicrope FA, Foster NR, Thibodeau SN, Marsoni S, Monges G, Labianca R, Kim GP, Yothers G, Allegra C, Moore MJ, Gallinger $\mathrm{S}$ and Sargent DJ: DNA mismatch repair status and colon cancer recurrence and survival in clinical trials of 5-fluorouracil-based adjuvant therapy. J Natl Cancer Inst 103: 863-875, 2011.

4 Goldstein J, Tran B, Ensor J, Gibbs P, Wong HL, Wong SF, Vilar E, Tie J, Broaddus R, Kopetz S, Desai J and Overman MJ: Multicenter retrospective analysis of metastatic colorectal cancer (CRC) with high-level microsatellite instability (MSI-H). Ann Oncol 25: 1032-1038, 2014.

5 Ogino $\mathrm{S}$ and Goel A: Molecular classification and correlates in colorectal cancer. J Mol Diagn 10: 13-27, 2008.

6 Ishikubo T, Nishimura Y, Yamaguchi K, Khansuwan U, Arai Y, Kobayashi T, Ohkura Y, Hashiguchi Y, Tanaka Y and Akagi K: The clinical features of rectal cancers with high-frequency microsatellite instability (MSI-H) in Japanese males. Cancer Lett 216: 55-62, 2004.

7 Lochhead P, Kuchiba A, Imamura Y, Liao X, Yamauchi M, Nishihara R, Qian ZR, Morikawa T, Shen J, Meyerhardt JA, Fuchs CS and Ogino S: Microsatellite instability and BRAF mutation testing in colorectal cancer prognostication. $\mathrm{J}$ Natl Cancer Inst 105: 1151-1156, 2013.

8 Kadowaki S, Kakuta M, Takahashi S, Takahashi A, Arai Y, Nishimura Y, Yatsuoka T, Ooki A, Yamaguchi K, Matsuo K, Muro $\mathrm{K}$ and Akagi $\mathrm{K}$ : Prognostic value of $K R A S$ and $B R A F$ mutations in curatively resected colorectal cancer. World $\mathrm{J}$ Gastroenterol 21: 1275-1283, 2015.

9 Nitsche U, Friess H, Agha A, Angele M, Eckel R, Heitland W, Jauch K-W, Krenz D, Nüssler N, Rau H-G, Ruppert R, Schubert Fritschle G, Wilhelm D, Werner J and Engel J: Prognosis of 
mucinous and signet-ring cell colorectal cancer in a populationbased cohort. J Cancer Res Clin Oncol 142: 2357-2366, 2016.

10 Ooki A, Akagi K, Yatsuoka T, Asayama M, Hara H, Takahashi A, Kakuta M, Nishimura Y and Yamaguchi K: Combined microsatellite instability and BRAF gene status as biomarkers for adjuvant chemotherapy in stage III colorectal cancer. J Surg Oncol 110: 982-988, 2014

11 Des Guetz G, Schischmanoff O, Nicolas P, Perret GY, Morere JF and Uzzan B: Does microsatellite instability predict the efficacy of adjuvant chemotherapy in colorectal cancer? A systematic review with meta-analysis. Eur J Cancer 45: 1890-1896, 2009.

12 Klingbiel D, Saridaki Z, Roth AD, Bosman FT, Delorenzi M and Tejpar S: Prognosis of stage II and III colon cancer treated with adjuvant 5-fluorouracil or FOLFIRI in relation to microsatellite status: results of the PETACC-3 trial. Ann Oncol 26: 126-132, 2015.

13 Venderbosch S, Nagtegaal ID, Maughan TS, Smith CG, Cheadle JP, Fisher D, Kaplan R, Quirke P, Seymour MT, Richman SD, Meijer GA, Ylstra B, Heideman DA, de Haan AF, Punt CJ and Koopman M: Mismatch repair status and BRAF mutation status in metastatic colorectal cancer patients: a pooled analysis of the CAIRO, CAIRO2, COIN and FOCUS studies. Clin Cancer Res 20: 5322-5330, 2014.

14 Smith CG, Fisher D, Claes B, Maughan TS, Idziaszczyk S, Peuteman G, Harris R, James MD, Meade A, Jasani B, Adams RA, Kenny S, Kaplan R, Lambrechts D and Cheadle JP: Somatic profiling of the epidermal growth factor receptor pathway in tumors from patients with advanced colorectal cancer treated with chemotherapy $+/-$ cetuximab. Clin Cancer Res 19: 4104-4113, 2013.

15 Le DT, Uram JN, Wang H, Bartlett BR, Kemberling H, Eyring AD, Skora AD, Luber BS, Azad NS, Laheru D, Biedrzycki B, Donehower RC, Zaheer A, Fisher GA, Crocenzi TS, Lee JJ, Duffy SM, Goldberg RM, de la Chapelle A, Koshiji M, Bhaijee F, Huebner T, Hruban RH, Wood LD, Cuka N, Pardoll DM, Papadopoulos N, Kinzler KW, Zhou S, Cornish TC, Taube JM, Anders RA, Eshleman JR, Vogelstein B and Diaz LA Jr.: PD-1 blockade in tumors with mismatch-repair deficiency. N Engl J Med 372: 2509-2520, 2015.

16 TNM Classification of Malignant Tumours (Seventh Edition). New York: Wiley-Blackwell, Inc., 2010.

17 Akagi K, Uchibori R, Yamaguchi K, Kurosawa K, Tanaka Y and Kozu T: Characterization of a novel oncogenic $K-R A S$ mutation in colon cancer. Biochem Biophys Res Commun 352: 728-732, 2007.

18 Ogura T, Kakuta M, Yatsuoka T, Nishimura Y, Sakamoto H, Yamaguchi K, Tanabe M, Tanaka Y and Akagi K: Clinicopathological characteristics and prognostic impact of colorectal cancers with NRAS mutations. Oncol Rep 32: 50-56, 2014.

19 Asaka S, Arai Y, Nishimura Y, Yamaguchi K, Ishikubo T, Yatsuoka T, Tanaka Y and Akagi K: Microsatellite instability-low colorectal cancer acquires a KRAS mutation during the progression from Dukes' A to Dukes' B. Carcinogenesis 30: 494-499, 2009.

20 Van Cutsem E, Kohne CH, Lang I, Folprecht G, Nowacki MP, Cascinu S, Shchepotin I, Maurel J, Cunningham D, Tejpar S, Schlichting M, Zubel A, Celik I, Rougier P and Ciardiello F: Cetuximab plus irinotecan, fluorouracil and leucovorin as firstline treatment for metastatic colorectal cancer: updated analysis of overall survival according to tumor KRAS and BRAF mutation status. J Clin Oncol 29: 2011-2019, 2011.
21 Di Nicolantonio F, Martini M, Molinari F, Sartore-Bianchi A, Arena S, Saletti P, De Dosso S, Mazzucchelli L, Frattini M, Siena $S$ and Bardelli A: Wild-type BRAF is required for response to panitumumab or cetuximab in metastatic colorectal cancer. J Clin Oncol 26: 5705-5712, 2008.

22 Ahn TS, Jeong D, Son MW, Jung H, Park S, Kim H, Bae SB, Kim HJ, Jeon YW, Lee MS and Baek MJ: The BRAF mutation is associated with the prognosis in colorectal cancer. J Cancer Res Clin Oncol 140: 1863-1871, 2014.

23 Phipps AI, Limburg PJ, Baron JA, Burnett-Hartman AN, Weisenberger DJ, Laird PW, Sinicrope FA, Rosty C, Buchanan DD, Potter JD and Newcomb PA: Association between molecular subtypes of colorectal cancer and patient survival. Gastroenterology 148: 77-87 e72, 2015.

24 Nakaji Y, Oki E, Nakanishi R, Ando K, Sugiyama M, Nakashima Y, Yamashita N, Saeki H, Oda Y and Maehara Y: Prognostic value of BRAF V600E mutation and microsatellite instability in Japanese patients with sporadic colorectal cancer. J Cancer Res Clin Oncol doi:10.1007/s00432-016-2275-4, 2016

25 Gryfe R, Kim H, Hsieh ET, Aronson MD, Holowaty EJ, Bull SB, Redston $\mathrm{M}$ and Gallinger S: Tumor microsatellite instability and clinical outcome in young patients with colorectal cancer. $\mathrm{N}$ Engl J Med 342: 69-77, 2000.

26 Oh BY, Huh JW, Park YA, Cho YB, Yun SH, Kim HC, Lee WY and Chun HK: Prognostic factors in sporadic colon cancer with high-level microsatellite instability. Surgery 159: 1372-1381, 2016.

27 Guinney J, Dienstmann R, Wang X, de Reynies A, Schlicker A, Soneson C, Marisa L, Roepman P, Nyamundanda G, Angelino P, Bot BM, Morris JS, Simon IM, Gerster S, Fessler E, De Sousa EMF, Missiaglia E, Ramay H, Barras D, Homicsko K, Maru D, Manyam GC, Broom B, Boige V, Perez-Villamil B, Laderas T, Salazar R, Gray JW, Hanahan D, Tabernero J, Bernards R, Friend SH, Laurent-Puig P, Medema JP, Sadanandam A, Wessels L, Delorenzi M, Kopetz S, Vermeulen L and Tejpar S: The consensus molecular subtypes of colorectal cancer. Nat Med 21: 1350-1356, 2015.

28 Vatandoust S, Price TJ and Karapetis CS: Colorectal cancer: Metastases to a single organ. World J Gastroenterol 21: 1176711776, 2015.

29 Tran B, Kopetz S, Tie J, Gibbs P, Jiang ZQ, Lieu CH, Agarwal A, Maru DM, Sieber O and Desai J: Impact of BRAF mutation and microsatellite instability on the pattern of metastatic spread and prognosis in metastatic colorectal cancer. Cancer 117: 4623-4632, 2011.

30 Birgisson $\mathrm{H}$, Edlund $\mathrm{K}$, Wallin U, Påhlman L, Kultima H, Mayrhofer M, Micke P, Isaksson A, Botling J, Glimelius B and Sundström M: Microsatellite instability and mutations in $B R A F$ and $K R A S$ are significant predictors of disseminated disease in colon cancer. BMC cancer 15: 125-125, 2015.

31 Chang SC, Lin PC, Yang SH, Wang HS, Liang WY and Lin JK: Taiwan hospital-based detection of Lynch syndrome distinguishes 2 types of microsatellite instabilities in colorectal cancers. Surgery 147: 720-728, 2010.

32 Oh JR, Kim DW, Lee HS, Lee HE, Lee SM, Jang JH, Kang SB, $\mathrm{Ku}$ JL, Jeong SY and Park JG: Microsatellite instability testing in Korean patients with colorectal cancer. Fam Cancer 11: 459466, 2012.

Received October 28, 2016

Revised November 14, 2016 Accepted November 15, 2016 